Article

\title{
Efficacy and Safety of OnabotulinumtoxinA 400 Units in Patients with Post-Stroke Upper Limb Spasticity: Final Report of a Randomized, Double-Blind, Placebo-Controlled Trial with an Open-Label Extension Phase
}

\author{
Masahiro Abo ${ }^{1, *(\mathbb{D})}$, Takashi Shigematsu ${ }^{2,+}{ }^{\dagger}$, Hiroyoshi Hara ${ }^{3, \ddagger}$, Yasuko Matsuda ${ }^{4, *}$, \\ Akinori Nimura ${ }^{4}$, Yoshiyuki Yamashita ${ }^{4, *}$ and Kaoru Takahashi ${ }^{4}$ \\ 1 Department of Rehabilitation Medicine, The Jikei University School of Medicine, Tokyo 105-8461, Japan \\ 2 Department of Rehabilitation Medicine, Seirei Hamamatsu City Rehabilitation Hospital, Shizuoka 433-8511, \\ Japan; t-shige@sis.seirei.or.jp \\ 3 Department of Rehabilitation Medicine, Kikyogahara Hospital, Nagano 399-6461, Japan; \\ hhara448@icloud.com \\ 4 GlaxoSmithKline K.K., Tokyo 107-0052, Japan; akinori.x.nimura@gsk.com (A.N.); \\ kaoru.2.takahashi@gsk.com (K.T.) \\ * Correspondence: abo@jikei.ac.jp (M.A.); yasuko.2.matsuda@gsk.com (Y.M.); \\ yoshiyuki.2.yamashita@gsk.com (Y.Y.) \\ † Current affiliation: Department of Rehabilitation Medicine, Seirei Awaji Hospital, Hyogo 656-2306, Japan. \\ $\ddagger$ Current affiliation: Neurorehabilitation Center, Ainomiyako Neurosurgery Hospital, Osaka 538-0044, Japan.
}

Received: 24 December 2019; Accepted: 14 February 2020; Published: 18 February 2020

\begin{abstract}
In many countries, 400 units (U) is the maximum dose of onabotulinumtoxinA available to treat upper limb spasticity, but few studies have demonstrated the optimal use of this dose. In the double-blind phase of this randomized, controlled trial, we compared the efficacy and safety of 400 vs. $240 \mathrm{U}$ onabotulinumtoxinA in patients with post-stroke upper limb spasticity. Both groups received $240 \mathrm{U}$ onabotulinumtoxinA injected in the forearm. An additional $160 \mathrm{U}$ onabotulinumtoxinA (400 U group) or placebo (240 U group) was injected in the elbow flexors. Both groups showed similar muscle tone reduction in the wrist, fingers, and thumb; muscle tone reduction in the elbow flexors was greater in the group treated with onabotulinumtoxinA ( $400 \mathrm{U}$ group) compared to placebo ( $240 \mathrm{U}$ group). Functional disabilities improved in both groups. No substantial difference was found in safety profiles. In the subsequent open-label phase, all participants received repeat injections of $400 \mathrm{U}$ onabotulinumtoxinA (target muscles and doses per muscle determined by the physician). Similar efficacy and safety outcomes, as with the $400 \mathrm{U}$ group in the double-blind phase, were confirmed. This final report demonstrates that injection of onabotulinumtoxinA $400 \mathrm{U}$ relieves muscle tone in a wide range of areas and improves functional disabilities; generally, it was well-tolerated, and no new safety concerns were identified. The dosing data in the open-label phase will inform optimal use of onabotulinumtoxinA in clinical practice (ClinicalTrials.gov: NCT03261167).
\end{abstract}

Keywords: botulinum toxin; stroke; upper limb spasticity; randomized controlled trial

Key Contribution: This trial has demonstrated the efficacy and safety of onabotulinumtoxinA $400 \mathrm{U}$ in comparison with $240 \mathrm{U}$, and how it can be used effectively to treat upper limb spasticity in clinical practice. 


\section{Introduction}

Spasticity has been historically defined as a motor disorder characterized by a velocity-dependent increase in tonic stretch reflexes (muscle tone) [1]. In clinical practice, the term is often used to describe a wide range of disabling symptoms resulting from muscle overactivity, including continuous muscle stiffness, involuntary contractions, and pain and discomfort, which can negatively impact patient quality of life [2].

Botulinum toxin type A (BoNT/A) is included as part of standard treatment modalities for patients with spasticity [3]. Its efficacy and safety for post-stroke upper limb spasticity have been demonstrated in randomized controlled trials [4]. Several formulations of BoNT/A are commercially available. However, these are not interchangeable with one another due to the different clinical characteristics of each [5].

OnabotulinumtoxinA (BOTOX, Allergan plc, Irvine, CA, USA) is a BoNT/A formulation that has been approved and used in more than 90 countries for upper limb spasticity, with 400 units (U) stipulated as the maximum dose for this indication in many countries. In Japan, a phase 3 clinical trial was conducted to evaluate the efficacy and safety of $240 \mathrm{U}$ injected in forearm muscles [6], and the results of this trial led to the approval of $240 \mathrm{U}$ as the maximum dose for upper limb spasticity. In clinical practice, however, many patients require injections in elbow flexors (upper arm muscles) and/or shoulder adductors/internal rotators, which often necessitates doses higher than $240 \mathrm{U}$ in order to properly treat these additional muscles. Therefore, we conducted another phase 3 trial to evaluate the efficacy and safety of $400 \mathrm{U}$ as compared to $240 \mathrm{U}$, in order to obtain approval for the escalation of the maximum dose.

In the double-blind phase of this phase 3, randomized, placebo-controlled trial, we compared the efficacy and safety of onabotulinumtoxinA $400 \mathrm{vs.} 240 \mathrm{U}$ in patients with post-stroke upper limb spasticity. In the subsequent open-label phase, up to 3 injections of onabotulinumtoxinA $400 \mathrm{U}$ were given to all patients that met the re-treatment criteria, with the muscles injected (including shoulder adductors/internal rotators and forearm pronators) and doses per muscle determined at the discretion of the physician, to evaluate the efficacy and safety of repeat injections (Figure 1).

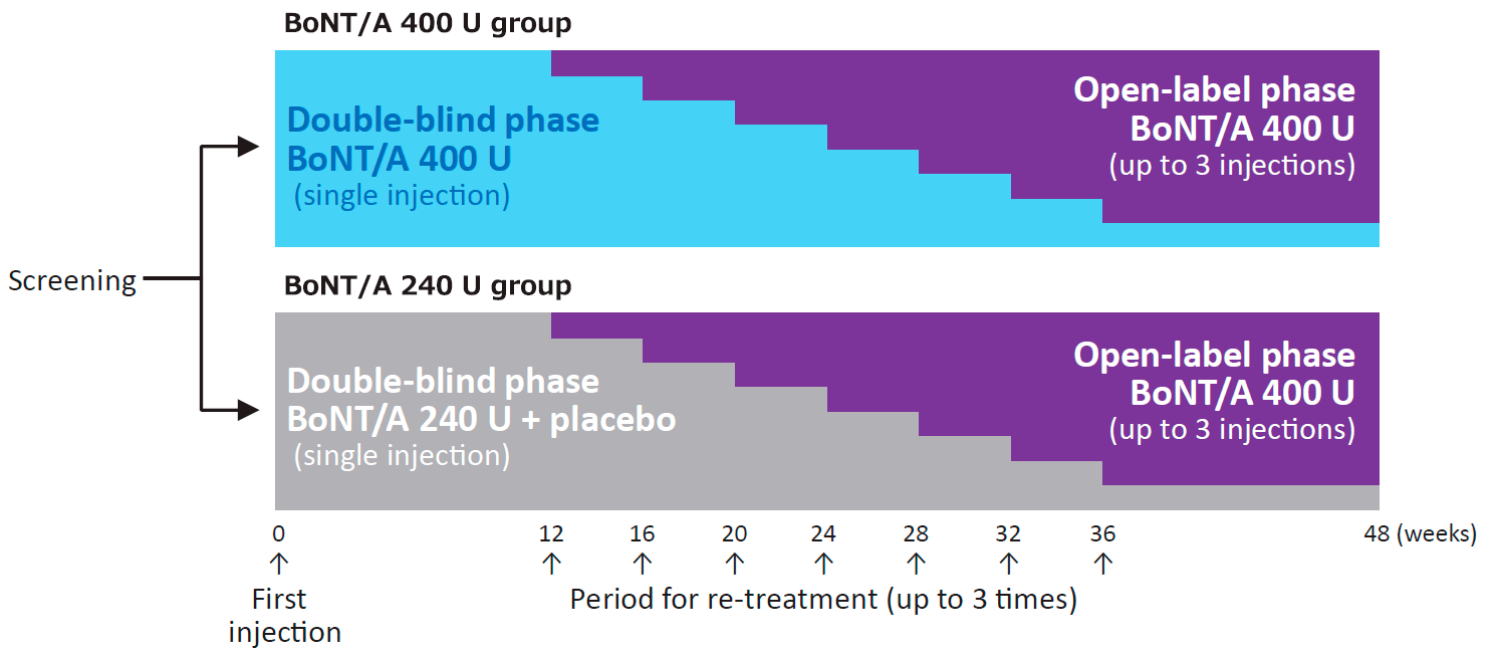

Figure 1. Study design. BoNT/A, botulinum toxin type A (onabotulinumtoxinA). Reproduced with permission from Abo, M., et al., Progress in Medicine, published by Life Science Co., Ltd., 2019 [in Japanese]. 


\section{Results}

\subsection{Patient Demographics and Clinical Characteristics}

Of 131 patients who were screened, 124 were randomized (61 in the $400 \mathrm{U}$ group; 63 in the $240 \mathrm{U}$ group) and received the first administration of the study drug. A total of 113 patients completed the 48-week study, while 11 patients ( 5 in the $400 \mathrm{U}$ group; 6 in the $240 \mathrm{U}$ group) withdrew from the trial. The primary reasons for withdrawal were withdrawal of consent (7 patients), adverse events (AEs) (3 patients), and meeting the criteria for discontinuation (1 patient) (Figure S1).

Patient demographics and clinical characteristics have been previously reported and are shown in Table 1. The proportion of male patients was slightly lower in the $400 \mathrm{U}$ group, otherwise no substantial differences were noted between treatment groups. The Modified Ashworth Scale (MAS) score at baseline in the elbow was 3 in 113 patients and 4 in 11 patients (which were coded as 4 and 5 , respectively, for tabulation). Of the 4 domains of the Disability Assessment Scale (DAS), "limb position" was selected most frequently as a principal therapeutic target (44 patients, 35\%), followed by "dressing" (35 patients, 28\%), "hygiene" (28 patients, 23\%), and "pain" (17 patients, 14\%). These proportions were similar between treatment groups.

Table 1. Patient demographics and clinical characteristics.

\begin{tabular}{cccc}
\hline Variables & $\begin{array}{c}\text { 400 U Group } \\
\mathbf{( N = 6 1 )}\end{array}$ & $\begin{array}{c}\mathbf{2 4 0} \text { U Group } \\
\mathbf{( N = 6 3 )}\end{array}$ & $\begin{array}{c}\text { Total } \\
(\mathbf{N}=\mathbf{1 2 4})\end{array}$ \\
\hline Sex (male) & $46(75 \%)$ & $53(84 \%)$ & $99(80 \%)$ \\
Age (years) & $57.1 \pm 9.90$ & $57.3 \pm 10.98$ & $57.2 \pm 10.42$ \\
Height (cm) & $166.2 \pm 8.94$ & $164.8 \pm 7.31$ & $165.5 \pm 8.15$ \\
Weight (kg) & $67.67 \pm 12.794$ & $66.13 \pm 10.667$ & $66.89 \pm 11.739$ \\
Elbow MAS score & $4.1 \pm 0.28$ & $4.1 \pm 0.30$ & $4.1 \pm 0.29$ \\
DAS principal therapeutic target score & $2.0 \pm 0.80$ & $1.9 \pm 0.87$ & $2.0 \pm 0.84$ \\
\hline
\end{tabular}

Number of patients (\%) or mean \pm standard deviation. DAS, Disability Assessment Scale; MAS, Modified Ashworth Scale. MAS scores of $0,1,1+, 2,3$, and 4 were coded as $0,1,2,3,4$, and 5 , respectively, for tabulation: $0=$ no increase in muscle tone; 5 = affected part(s) rigid in flexion or extension. Reproduced with permission from Abo, M., et al., Progress in Medicine, published by Life Science Co., Ltd., 2019 [in Japanese].

At the time of first injection of the study drug, participants were using several rehabilitation modalities including stretching/range of motion (ROM) exercise by 83 patients (67\%); muscle strengthening exercise by 41 patients (33\%); task-specific training by 29 patients (23\%); positioning aids by 16 patients (13\%); splinting/orthoses by 14 patients $(11 \%)$; and taping by 0 patients $(0 \%)$. No substantial difference was observed between groups regarding the use of these modalities.

\subsection{Efficacy}

During the double-blind phase, both groups received an injection of $240 \mathrm{U}$ onabotulinumtoxinA in the forearm (wrist, finger, and thumb flexors) and an additional injection of onabotulinumtoxinA $160 \mathrm{U}$ (400 U group) or placebo (240 U group) in the elbow flexors at week 0 (treatment cycle 1). At week 6 (primary endpoint), the proportion of patients with $\geq 1$-point reduction in the MAS score (responder rate) in the elbow was 68.9\% (42/61) in the $400 \mathrm{U}$ group and 50.8\% (32/63) in the $240 \mathrm{U}$ group. The difference between the groups was $18.1 \%$, with a $95 \%$ confidence interval of $1.1 \%$ to $35.0 \%$. The responder rates in the wrist (68.9\% for the $400 \mathrm{U}$ group; $81.0 \%$ for the $240 \mathrm{U}$ group), fingers (72.1\% for the $400 \mathrm{U}$ group; $81.0 \%$ for the $240 \mathrm{U}$ group), and thumb $(66.7 \%$ for the $400 \mathrm{U}$ group; $68.3 \%$ for the $240 \mathrm{U}$ group) were comparable between groups at week 6. During the double-blind phase through week 12, the elbow MAS score was consistently lower in the $400 \mathrm{U}$ group compared with the $240 \mathrm{U}$ group (Table S1), and the change in score (degree of improvement) was consistently greater in the $400 \mathrm{U}$ group vs. the $240 \mathrm{U}$ group. Conversely, score reductions in the wrist, fingers, and thumb were comparable between groups (Table S1). 
In the open-label phase, in which all patients received $400 \mathrm{U}$ onabotulinumtoxinA (up to 3 treatment cycles separated by $\geq 12$ weeks), responder rates (proportions of patients demonstrating $\geq 1$-point reduction in the MAS score) were similar at week 6 in each treatment cycle (treatment cycles 2,3, and 4, respectively) at the elbow $(77.6 \%, 73.4 \%$, and $77.8 \%)$, wrist $(84.8 \%, 80.0 \%$, and $84.3 \%)$, fingers $(77.5 \%$, $74.8 \%$, and $81.8 \%$ ), and thumb (73.6\%, $68.5 \%$, and $79.6 \%)$ as in the $400 \mathrm{U}$ group during the double-blind phase. Degrees of improvement in MAS scores for these joints were similar to those found in the 400 group after the first injection, with the greatest improvement noted from week 2 through week 6 (Figure 2). The MAS scores for the forearm pronation and shoulder adduction/internal rotation also decreased in the open-label phase (Table S1).

(a) Elbow

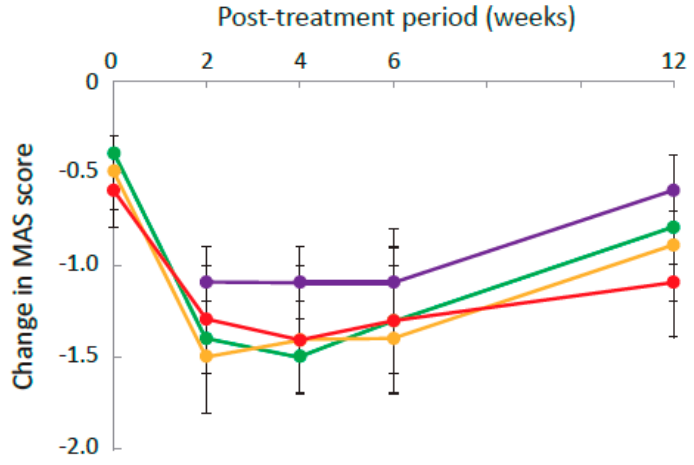

(c) Fingers

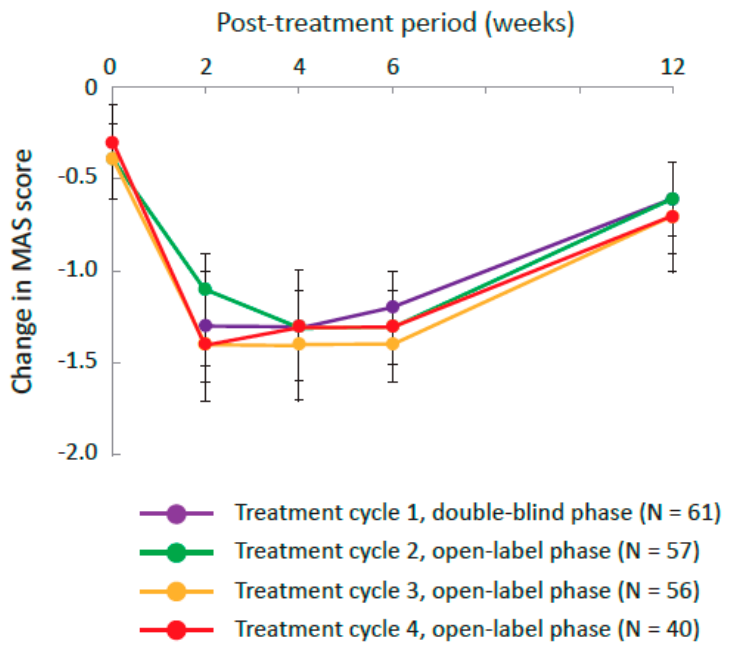

(b) Wrist

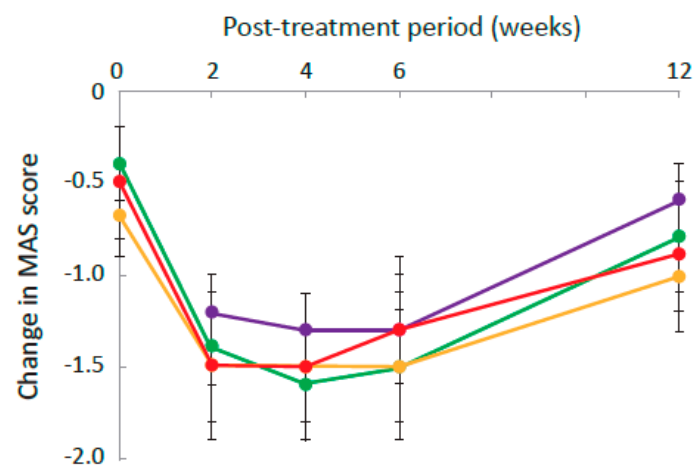

(d) Thumb

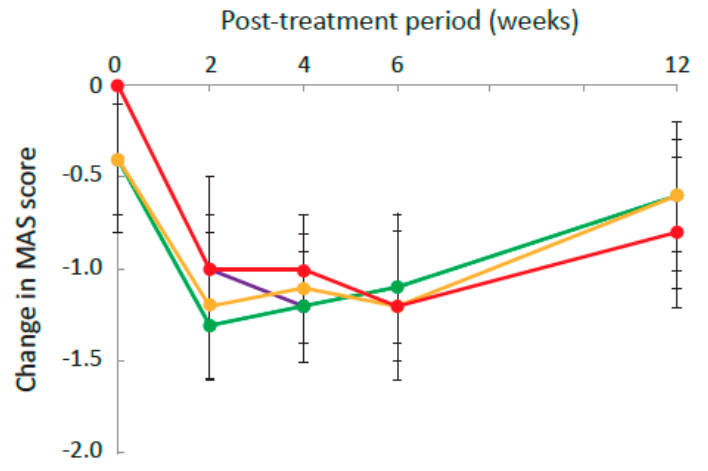

Adjusted mean $\pm 95 \% \mathrm{Cl}$

Figure 2. Change from baseline in the Modified Ashworth Scale (MAS) scores across all treatment cycles for the $400 \mathrm{U}$ group at (a) elbow, (b) wrist, (c) fingers, and (d) thumb. Treatment cycles are represented as: treatment cycle 1 (double-blind phase, purple), treatment cycle 2 (open-label phase, green), treatment cycle 3 (open-label phase, yellow), and treatment cycle 4 (open-label phase, red). CI, confidence interval.

In the double-blind phase (treatment cycle 1), the DAS principal therapeutic target score decreased from baseline through week 12 in both groups, showing an improvement in functional disabilities due to spasticity. The improvement was comparable between groups, but the mean (standard deviation (SD)) DAS score was numerically lower in the $400 \mathrm{U}$ group (1.4 (0.82) at week 12) compared with the $240 \mathrm{U}$ group (1.6 (0.89) at week 12). In the open-label phase, the DAS score decreased after each injection of $400 \mathrm{U}$ onabotulinumtoxinA (treatment cycles 2, 3, and 4), showing continued improvement in functional disabilities (Table 2). 
Table 2. Change from baseline in the DAS principal therapeutic target score across all treatment cycles for the $400 \mathrm{U}$ group.

\begin{tabular}{ccccccc}
\hline Treatment Cycles & Parameters & Week 0 & Week 2 & Week 4 & Week 6 & Week 12 \\
\hline $\begin{array}{c}\text { Double-blind phase } \\
\text { First injection }\end{array}$ & Mean & - & -0.6 & -0.6 & -0.7 & -0.6 \\
$\quad \mathrm{~N}=61)$ & $95 \% \mathrm{CI}$ & - & $(-0.8,-0.4)$ & $(-0.8,-0.5)$ & $(-0.9,-0.5)$ & $(-0.9,-0.4)$ \\
Open-label phase & & & & & \\
Second injection & Mean & -0.6 & -0.7 & -0.8 & -0.8 & -0.7 \\
$(\mathrm{~N}=57)$ & $95 \% \mathrm{CI}$ & $(-0.8,-0.4)$ & $(-0.9,-0.5)$ & $(-1.0,-0.6)$ & $(-1.0,-0.6)$ & $(-0.9,-0.5)$ \\
Third injection & Mean & -0.6 & -0.9 & -0.9 & -0.8 & -0.8 \\
$(\mathrm{~N}=56)$ & $95 \% \mathrm{CI}$ & $(-0.8,-0.4)$ & $(-1.1,-0.6)$ & $(-1.1,-0.6)$ & $(-1.1,-0.6)$ & $(-1.0,-0.5)$ \\
Fourth injection & Mean & -0.7 & -0.9 & -0.9 & -0.9 & -0.9 \\
$(\mathrm{~N}=40)$ & $95 \% \mathrm{CI}$ & $(-1.0,-0.4)$ & $(-1.2,-0.6)$ & $(-1.2,-0.6)$ & $(-1.1,-0.6)$ & $(-1.1,-0.6)$ \\
\hline
\end{tabular}

CI, confidence interval; DAS, Disability Assessment Scale.

All DAS domain scores slightly decreased (demonstrating improvement) from baseline through week 12 in both groups during the double-blind phase (Table S2). The improvement in "limb position" was slightly greater in the $400 \mathrm{U}$ group than in the $240 \mathrm{U}$ group, whereas improvements in the other domains were similar between groups. In the open-label phase, all DAS domain scores showed similar degrees of improvement as shown in the double-blind phase (Table S2).

Both in the double-blind phase and the open-label phase, the physician-assessed Clinical Global Impression of Change (CGI) showed positive scores (demonstrating improvement) at week 2. This improvement was sustained through week 6 and had attenuated at week 12 of each cycle (Table S3). The patient-assessed CGI followed a similar course of improvement and attenuation. No substantial difference was noted between groups whether CGI was assessed by the physician or the patient (Table S3).

\subsection{Safety}

In the first 12 weeks of the double-blind phase, AE rates were similar between groups (51\% (31/61) in the $400 \mathrm{U}$ group and $46 \%(29 / 63)$ in the $240 \mathrm{U}$ group). The most frequently reported AEs were nasopharyngitis $(11 \%(7 / 61)$ and $17 \%(11 / 63)$ in the $400 \mathrm{U}$ and $240 \mathrm{U}$ groups, respectively) and fall (11\% $(7 / 61)$ and 3\% (2/63) in the $400 \mathrm{U}$ and $240 \mathrm{U}$ groups, respectively). Fall and contusion had incidences at least $5 \%$ higher in the $400 \mathrm{U}$ group than the $240 \mathrm{U}$ group and arthralgia, muscle spasms, constipation, and subcutaneous hemorrhage occurred only in the $400 \mathrm{U}$ group (Table 3). None of these common AEs were considered by the physician to be related to the study drug.

Table 3. Common adverse events in the 12 weeks following the first injection (double-blind phase).

\begin{tabular}{ccc}
\hline Adverse Events & $\begin{array}{c}\text { 400 U Group } \\
\mathbf{( N = 6 1 )}\end{array}$ & $\begin{array}{c}\mathbf{2 4 0} \text { U Group } \\
\mathbf{( N = 6 3 )}\end{array}$ \\
\hline Total number of patients with adverse events & $31(51 \%)$ & $29(46 \%)$ \\
Nasopharyngitis & $7(11 \%)$ & $11(17 \%)$ \\
Fall & $7(11 \%)$ & $2(3 \%)$ \\
Contusion & $4(7 \%)$ & $1(2 \%)$ \\
Influenza & $2(3 \%)$ & $3(5 \%)$ \\
Insomnia & $3(5 \%)$ & $1(2 \%)$ \\
Back pain & $3(5 \%)$ & $1(2 \%)$ \\
Arthralgia & $1(2 \%)$ & $2(3 \%)$ \\
Muscle spasms & $2(3 \%)$ & 0 \\
Constipation & $2(3 \%)$ & 0 \\
Hemorrhage subcutaneous & $2(3 \%)$ & 0 \\
\hline
\end{tabular}

Number of patients (\%). Adverse events reported by more than one patient in either group are shown. Reproduced with permission from Abo, M., et al., Progress in Medicine, published by Life Science Co., Ltd., 2019 [in Japanese]. 
During the overall study period, the incidence of AEs was 80\% (49/61) in the $400 \mathrm{U}$ group and $83 \%$ (52/63) in the $240 \mathrm{U}$ group. The most frequently reported AEs overall were fall (25\% (15/61) and 17\% $(11 / 63)$ in the $400 \mathrm{U}$ and $240 \mathrm{U}$ groups, respectively) and nasopharyngitis (16\% (10/61) and 24\% (15/63) in the $400 \mathrm{U}$ and $240 \mathrm{U}$ groups, respectively). Other common AEs reported in $\geq 3 \%$ of patients in either group are shown in Table S4. None of these AEs were considered to be related to the study drug.

Two drug-related AEs as assessed by the physician occurred during the overall study period. Muscular weakness was experienced by one patient $(2 \%(1 / 61))$ in the $400 \mathrm{U}$ group during the double-blind phase, 8 days after the first treatment, and was considered to be local to the site of injection. Mild injection site swelling was experienced by one patient $(2 \%(1 / 63))$ in the 240 U group during the open-label phase, 2 days after the third injection (onabotulinumtoxinA $400 \mathrm{U}$ ) and resolved after 28 days.

Across the overall study period, the incidence of nonfatal serious AEs (SAEs) was 11\% (7/61) in the $400 \mathrm{U}$ group and 10\% (6/63) in the $240 \mathrm{U}$ group. Nonfatal SAEs occurring in 2 or more patients were pneumonia (one in each group), decreased activity (one in each group), and cerebral hemorrhage ( 2 in the $400 \mathrm{U}$ group). None of the nonfatal SAEs were considered to be related to the study drug. One fatal SAE, pneumonia, occurred during the double-blind phase in the $400 \mathrm{U}$ group, and was assessed as not related to the study drug.

Three AEs leading to study withdrawal occurred in the $400 \mathrm{U}$ group (5\% (3/61)), all of which were reported in the double-blind phase and assessed as SAEs not related to the study drug. The AEs included pneumonia (fatal) and cerebral hemorrhage during the double-blind phase, and Alzheimer's dementia that occurred 243 days after the first injection in the double-blind phase. No AEs leading to study withdrawal occurred in the $240 \mathrm{U}$ group. During the open-label phase, no patient discontinued due to AEs.

\subsection{Dosing in the Open-label Phase}

In the open-label phase, up to 3 injections of $400 \mathrm{U}$ onabotulinumtoxinA were given to all patients that met the re-treatment criteria. The muscles to be injected and doses per muscle were determined at the discretion of the physician. In total, 117 patients received injections of $400 \mathrm{U}$ in 311 treatment sessions across several joints (Table S5). The mean dose per muscle varied between $28.5 \mathrm{U}$ (flexor pollicis longus) and $74.0 \mathrm{U}$ (biceps brachii). Biceps brachii, brachialis, flexor carpi radialis, and flexor digitorum superficialis were among the most frequently injected muscles (Table 4; more details in Table S6).

Table 4. Muscles injected in the open-label phase.

\begin{tabular}{cccc}
\hline Muscles & No. of Patients & No. of Treatment Sessions & Mean Dose (95\% CI) \\
\hline All muscles combined & 117 & 311 & - \\
Pectoralis major & 84 & 195 & $56.2(51.5,60.8)$ \\
Latissimus dorsi & 36 & 69 & $45.8(38.4,53.1)$ \\
Teres major & 16 & 27 & $42.1(34.9,49.3)$ \\
Subscapularis & 9 & 17 & $42.7(33.9,51.4)$ \\
Biceps brachii & 116 & 296 & $74.0(70.4,77.6)$ \\
Brachialis & 110 & 261 & $45.3(42.6,47.9)$ \\
Brachioradialis & 87 & 168 & $41.6(38.9,44.2)$ \\
Pronator teres & 73 & 175 & $47.2(43.9,50.5)$ \\
Pronator quadratus & 4 & 8 & $45.3(15.4,75.2)$ \\
Flexor carpi radialis & 108 & 267 & $50.1(46.9,53.2)$ \\
Flexor carpi ulnaris & 94 & 213 & $42.4(40.1,44.6)$ \\
Flexor digitorum profundus & 85 & 200 & $48.8(45.0,52.6)$ \\
Flexor digitorum superficialis & 111 & 276 & $56.2(52.9,59.5)$ \\
Lumbricals & 22 & 46 & $39.6(31.1,48.1)$ \\
Flexor pollicis longus & 84 & 174 & $28.5(26.1,30.9)$ \\
Adductor pollicis & 80 & 166 & $28.9(26.0,31.8)$ \\
Opponens pollicis & 7 & 12 & - \\
\hline
\end{tabular}

The estimate for the mean dose in opponens pollicis was not obtained because of the small amount of data. CI, confidence interval. 
Some agonist muscles work in conjunction with each other to flex or extend a joint, therefore, the target muscles to be injected for the same deformity can vary from patient to patient. Table 5 shows combinations of muscles frequently injected in the open-label phase of this trial. In the treatment of shoulder adduction/internal rotation, for example, most patients received injections in pectoralis major, with or without an additional injection in latissimus dorsi or teres major. More treatment details are shown in Tables S7-S12.

Table 5. Combinations of muscles frequently injected in the open-label phase.

\begin{tabular}{|c|c|c|c|c|}
\hline Joints & Muscles & No. of Patients & No. of Treatment Sessions & $\begin{array}{c}\text { Mean Dose } \\
(95 \% \text { CI })\end{array}$ \\
\hline \multirow[t]{3}{*}{ Shoulder } & PM & 58 & 111 & $55.3(50.7,60.0)$ \\
\hline & $\mathrm{PM}+\mathrm{LD}$ & 30 & 54 & $101.0(84.8,117.1)$ \\
\hline & $\mathrm{PM}+\mathrm{TM}$ & 6 & 10 & - \\
\hline \multirow[t]{4}{*}{ Elbow } & $\mathrm{BB}+\mathrm{B}+\mathrm{BR}$ & 74 & 147 & $160.2(151.8,168.6)$ \\
\hline & $\mathrm{BB}+\mathrm{B}$ & 58 & 106 & $117.7(109.7,125.6)$ \\
\hline & BB & 25 & 28 & $89.6(81.2,98.1)$ \\
\hline & $\mathrm{BB}+\mathrm{BR}$ & 12 & 15 & $125.0(101.7,148.4)$ \\
\hline Forearm (pronation) & PT & 71 & 167 & $47.1(43.8,50.5)$ \\
\hline \multirow[t]{2}{*}{ Wrist } & $\mathrm{FCR}+\mathrm{FCU}$ & 93 & 210 & $91.2(86.0,96.5)$ \\
\hline & FCR & 36 & 57 & $53.7(50.2,57.2)$ \\
\hline \multirow[t]{5}{*}{ Fingers } & FDP + FDS & 75 & 177 & $102.9(95.9,109.9)$ \\
\hline & FDS & 37 & 66 & $60.7(53.8,67.5)$ \\
\hline & $\mathrm{FDP}+\mathrm{FDS}+\mathrm{LI}$ & 10 & 18 & $136.6(118.4,154.9)$ \\
\hline & FDS + LI & 9 & 15 & $110.3(92.5,128.1)$ \\
\hline & LI & 6 & 10 & - \\
\hline \multirow[t]{3}{*}{ Thumb } & $\mathrm{FPL}+\mathrm{AP}$ & 55 & 111 & $52.2(46.9,57.6)$ \\
\hline & FPL & 38 & 56 & $30.6(26.9,34.4)$ \\
\hline & $\mathrm{AP}$ & 33 & 48 & $37.8(32.5,43.0)$ \\
\hline
\end{tabular}

$\mathrm{AP}$, adductor pollicis; $\mathrm{B}$, brachialis; $\mathrm{BB}$, biceps brachii; $\mathrm{BR}$, brachioradialis; $\mathrm{CI}$, confidence interval; $\mathrm{FCR}$, flexor carpi radialis; FCU, flexor carpi ulnaris; FDP, flexor digitorum profundus; FDS, flexor digitorum superficialis; FPL, flexor pollicis longus; LD, latissimus dorsi; LI, lumbricals; PM, pectoralis major; PT, pronator teres; TM, teres major. Some estimates were not obtained because of the small amount of data.

We also performed an exploratory analysis to see whether the severity of spasticity affected the dose injected in each muscle. In most muscles, patients with a MAS score of 3 or 4 (which were coded as 4 and 5, respectively, for tabulation) prior to injection received higher doses of onabotulinumtoxinA compared with those with a lower MAS score, but the differences between the groups were not large (Table S13).

\section{Discussion}

The responder rates in the elbow at week 6 after the first injection of the study drug (primary endpoint) were $68.9 \%$ in the $400 \mathrm{U}$ group and $50.8 \%$ in the $240 \mathrm{U}$ group, both of which were extremely close to the values assumed prior to the initiation of the trial (see "Materials and Methods"). Given that the patients in the $240 \mathrm{U}$ group did not receive onabotulinumtoxinA in their elbow flexors, it may seem unexpected that half of them showed a response; however, studies have shown that injecting saline may relieve muscle overactivity [6], and rehabilitation therapy was not prohibited during the trial, which may have contributed to the elbow tone reduction in the $240 \mathrm{U}$ group. Furthermore, injecting $240 \mathrm{U}$ in the forearm muscles might have alleviated the overactivity of elbow flexors, as was suggested in a report using median nerve block [7]. Regarding the selection of elbow flexors for injection, biceps brachii tends to be the first choice among the elbow flexors in the treatment of elbow flexion deformity [8,9], whereas other studies recommend injecting brachialis instead [10,11]. However, based on the results of this trial, which demonstrated a clinically significant improvement in approximately $70 \%$ of patients when the majority of those treated in the elbow received treatment in a combination of flexors, injecting multiple elbow flexors may be worth considering. 
In joints other than the elbow, the MAS assessment showed comparable improvement in the $400 \mathrm{U}$ and 240 U groups, which was not surprising considering that both groups received the same treatment in the forearm muscles. Similar improvement was also observed for the DAS assessment in both groups, and little between-group differences were observed except for "limb position." The injection of $240 \mathrm{U}$ BoNT/A in the forearm muscles has been shown to improve the DAS [6]. In our study, the ability of this scale to detect between-group differences may have been limited, because DAS does not assess outcomes directly related to elbow flexion deformities [12]. The same presumption may apply to the results of the CGI, which is a subjective assessment of a broad range of conditions by the physician and the patient.

Regarding the safety assessment, no substantial difference was observed between groups in the incidence of AEs, but the $400 \mathrm{U}$ group demonstrated a slightly more frequent incidence of falls and contusion after the first injection. It is widely known that post-stroke patients have a high risk of falls, and the occurrence of falls per se was not a surprising finding in this trial of elderly patients with hemiplegia and concurrent lower limb spasticity. Injections in the upper limb have been reported to improve walking speed and/or body balance $[13,14]$, and these effects might have affected the incidence of falls due to increased activity, but the results in the open-label phase do not support this hypothesis, as the rate of falls decreased across both treatment groups. In the treatment of upper limb spasticity, attention should be paid to the possibility of change in gross motor functions, but we believe this possibility does not pose a major issue when physicians select treatment modalities.

In this trial, up to 3 injections of $400 \mathrm{U}$ were evaluated during the open-label extension phase, and improvement of muscle tone and functional disabilities were repeatedly demonstrated, with no attenuation of efficacy. Repeat injections of $400 \mathrm{U}$ were generally well-tolerated, and there were no signs of increasing incidence of AEs. Efficacy and safety were demonstrated in an environment similar to real-world clinical practice, where target muscles and doses per muscle were determined at the discretion of the physician. To the best of our knowledge, this is the first study to show how onabotulinumtoxin $\mathrm{A} 400 \mathrm{U}$ can be distributed in a variety of upper limb muscles. The dosing data in the open-label phase, including doses per muscle and combinations of muscle frequently injected, will provide valuable information when considering how to use $400 \mathrm{U}$ effectively in clinical practice.

This trial has several limitations inherent in a comparative study. In the double-blind phase, injections in shoulder adductors/internal rotators and forearm pronators were prohibited, thus limiting the individualized optimization of botulinum toxin treatment. Furthermore, as the regimen and frequency/intensity of concomitant rehabilitation therapy could not be changed during the double-blind phase, it was difficult to conduct a combination therapy approach best suited for each patient's post-treatment conditions. In the open-label phase, on the other hand, all patients received $400 \mathrm{U}$, and there was no control group.

\section{Conclusions}

The results of this randomized, double-blind, placebo-controlled trial demonstrated that injection of onabotulinumtoxinA $400 \mathrm{U}$ relieves muscle tone in a wide range of areas, and improves functional disabilities; generally, it was well-tolerated, and no new safety concerns were identified. The dosing data in the open-label phase of this trial will inform optimal use of onabotulinumtoxinA in clinical practice.

\section{Materials and Methods}

\subsection{Study Design}

This was a phase 3, multicenter, randomized, double-blind, placebo-controlled clinical trial, conducted at 38 medical institutions in Japan from August 2017 through January 2019. The aim of the study was to evaluate the efficacy and safety of onabotulinumtoxinA $400 \mathrm{U}$ as compared with $240 \mathrm{U}$ for upper limb spasticity in post-stroke patients (ClinicalTrials.gov: NCT03261167). 
The study was conducted in compliance with the Declaration of Helsinki, the Ministerial Ordinance on Good Clinical Practice (GCP) for Drugs, and other relevant laws and regulations. The trial protocol was approved by the institutional review board for all 38 medical institutions including the Jikei University School of Medicine (approved by the Jikei University Hospital IRB for Medicinal Products on August 15, 2017), Seirei Hamamatsu City Rehabilitation Hospital (approved by Nakameguro Atlas Clinic IRB on July 5, 2017), and Kikyogahara Hospital (approved by Sugiura Clinic IRB on August 18, 2017). Written informed consent was obtained from all patients.

The study design is shown in Figure 1. The trial consisted of a screening phase (up to 4 weeks), a double-blind phase (minimum of 12 weeks), and an open-label phase (maximum of 36 weeks). In the double-blind phase, patients were randomly allocated in a 1:1 ratio to either $400 \mathrm{U}$ onabotulinumtoxinA or $240 \mathrm{U}$ onabotulinumtoxinA. They received a randomization number at registration based on a computer-generated randomization allocation table. The investigator, study staff at the site, and patient were blinded to the study treatment allocated to each individual patient during the overall study period. The sponsor personnel were unblinded after the database freeze for the interim analysis of the first 24 weeks, and had access to the subject level data. Patients who met the re-treatment criteria after week 12 entered the open-label phase and received $400 \mathrm{U}$ onabotulinumtoxinA up to 3 times, with a minimum interval of 12 weeks between injections. Results in the double-blind phase have been published elsewhere as an interim report (Abo, M., et al., Prog. Med. 2019, 39, 1021-1029, written in Japanese).

\subsection{Patients and Therapeutic Interventions}

Male or female patients (aged 20-80 years) with upper limb spasticity due to stroke were eligible if they had at least a 3-month interval since the most recent stroke, a history of prior treatment with onabotulinumtoxinA $240 \mathrm{U}$, and a sufficient degree of spasticity to warrant administration of onabotulinumtoxinA $400 \mathrm{U}$. Their MAS scores had to be at least 3 in the elbow and at least 2 in the wrist or fingers. Exclusion criteria included the presence of bilateral spasticity and fixed contracture in the upper limb to be treated.

At the start of the double-blind phase (week 0), patients in both groups received an injection of $240 \mathrm{U}$ in 6 forearm muscles (flexor carpi radialis, flexor carpi ulnaris, flexor digitorum profundus, flexor digitorum superficialis, flexor pollicis longus, and adductor pollicis). At the same time, an additional injection of onabotulinumtoxinA $160 \mathrm{U}$ (400 U group) or placebo ( $240 \mathrm{U}$ group) was given, in 3 elbow flexors (biceps brachii, brachialis, and brachioradialis). The recommended doses were $50 \mathrm{U}$ each for flexor carpi radialis, flexor carpi ulnaris, flexor digitorum profundus, and flexor digitorum superficialis; $20 \mathrm{U}$ each for flexor pollicis longus and adductor pollicis; $70 \mathrm{U}$ for biceps brachii; $45 \mathrm{U}$ each for brachialis and brachioradialis, but doses could be adjusted across muscles at the physician's discretion based on the severity of symptoms, as long as the total dose was $240 \mathrm{U}$ in the forearm muscles and $160 \mathrm{U}$ in the elbow flexors. If the patient had no symptoms in the thumb and $240 \mathrm{U}$ could be divided among the other forearm muscles, the thumb muscles did not need to be treated. The study drug was reconstituted with $2 \mathrm{~mL}$ normal saline per $100 \mathrm{U}$. In order to ensure precise localization of muscles, use of guidance such as electromyography, electrical stimulation, or ultrasonography was recommended.

Concomitant use of centrally acting muscle relaxants was allowed if the patient had been using them before participating in the trial, but the dose was not to be changed from the screening phase until the completion of the double-blind phase. Concomitant use of other botulinum toxin formulations and peripherally acting muscle relaxants (such as dantrolene), nerve blocks with phenol or ethanol, and surgical interventions for the upper limb were prohibited from the screening phase until the completion of the trial. In the double-blind phase, the regimen and frequency/intensity of concurrent rehabilitation therapy were not to be changed.

Entry criteria for the open-label phase included a MAS score of at least 2 in the elbow, and at least 2 in the wrist or fingers, and no drug-related SAE during the double-blind phase. In the open-label phase, patients received $400 \mathrm{U}$ of onabotulinumtoxinA up to 3 times with a minimum interval of 
12 weeks between injections. Target muscles were selected from a list of 17 muscles including forearm pronators and shoulder adductors/internal rotators (Table 4). The dose for each of these muscles was determined by the physician, who was provided with a list of recommended doses based on clinical guidelines as reference [15].

\subsection{Evaluation}

The MAS, DAS, and CGI were used for efficacy assessment. Evaluation was performed at weeks $0,2,4,6$, and 12. Efficacy analysis included all patients who received the study drug and had at least one assessment of efficacy.

The MAS is a rating scale widely used for spasticity assessment in clinical practice, which measures the degree of resistance to passive joint movement at rest on a scale of 6 levels, from 0 (no increase in muscle tone) to 4 (affected part[s] rigid in flexion or extension) [16]. In this trial, the primary endpoint was the proportion of patients with $\geq 1$-point reduction in the elbow MAS score at week 6 after the first injection. In the tabulation of MAS scores, scores of $0,1,1+, 2,3$, and 4 were coded as $0,1,2,3,4$, and 5 , respectively.

The DAS is a rating scale to assess the degree of upper limb functional disabilities due to spasticity, which measures 4 domains ("hygiene," "pain," "dressing," and "limb position") on a scale of 4 levels, from 0 (no disability) to 3 (severe disability) [12]. Before the administration of the study drug, the physician and the patient had a discussion and selected one of the 4 domains as the principal therapeutic target.

The CGI is a global rating scale used by the physician and the patient independently to measure clinical meaningfulness of changes in symptoms on a scale of 9 levels, from -4 (very much worsened) to +4 (very much improved). At the time of assessment by the physician, the patient's clinical symptoms, AEs, and therapeutic effects to the elbow, wrist, and fingers were considered.

Safety assessment included all patients who received the study drug. AEs were documented throughout the study. Safety endpoints included AEs, vital signs (blood pressure, pulse rate, body temperature), and laboratory tests (hematology, clinical chemistry, urinalysis).

\subsection{Statistical Analysis}

Approximately 120 patients (60 per group) were planned to be enrolled to confirm that the responder rate based on the elbow MAS score in the $400 \mathrm{U}$ group would exceed that in the $240 \mathrm{U}$ group. Assuming that the responder rate in the $400 \mathrm{U}$ group and the $240 \mathrm{U}$ group in this study would be $70.0 \%$ and $50.0 \%$, respectively, on the basis of the results of a phase 3 trial conducted in the US [17], the probability of the responder rate in the $400 \mathrm{U}$ group exceeding that in the $240 \mathrm{U}$ group as the point estimate was calculated to be $98 \%$ or more if 60 patients were enrolled in each group.

The between-group difference and 95\% confidence interval were calculated for the primary endpoint (responder rate in the elbow at week 6 after the first injection). Descriptive statistics were determined for the secondary endpoints (MAS scores and their changes from baseline in the elbow, wrist, fingers, and thumb; DAS principal therapeutic target scores and their changes from baseline). Changes in MAS scores and DAS principal therapeutic target scores were analyzed using the mixed model for repeated measures (MMRM). This model included treatment, visit, and treatment-by-visit interaction as categorical fixed effect, and baseline value and baseline-by-visit interaction as continuous fixed effect. Descriptive statistics were determined for DAS domain scores and the CGI by the physician and the patient. Regarding the exposure to onabotulinumtoxinA in the open-label phase, a post hoc analysis was performed using a negative binomial model with only intercept term to calculate the mean injection rate for each joint and muscle, and a mixed model with only intercept term to calculate descriptive statistics for dosages per joint and muscle. Furthermore, a mixed model with intercept and a MAS score $(\geq 3$ or $<3$ ) prior to the associated dose as a categorical variable was used to evaluate an impact of the prior MAS score on the next dose per muscle. Statistical analyses were performed using SAS version 9.4 (SAS Institute Inc, Cary, NC, USA). 
Supplementary Materials: The following are available online at http://www.mdpi.com/2072-6651/12/2/127/s1, Figure S1: Patient disposition; Table S1: MAS scores in the double-blind and open-label phases; Table S2: Change from baseline in DAS scores in the double-blind and open-label phases; Table S3: CGI in the double-blind and open-label phases; Table S4: Common adverse events during the overall study period (48 weeks); Table S5: Joints treated in the open-label phase; Table S6: Muscles injected in the open-label phase; Table S7: Muscles injected to treat shoulder adduction/internal rotation; Table S8: Muscles injected to treat elbow flexion; Table S9: Muscles injected to treat forearm pronation; Table S10: Muscles injected to treat wrist flexion; Table S11: Muscles injected to treat finger flexion; Table S12: Muscles injected to treat thumb flexion/adduction; Table S13: Mean doses per muscle separated by the MAS score prior to injection.

Data Sharing Statement: Anonymized individual participant data and study documents can be requested for further research from www.clinicalstudydatarequest.com.

Author Contributions: Conceptualization, Y.M., A.N., Y.Y. and K.T.; methodology, Y.M., A.N., Y.Y. and K.T.; software, A.N.; validation, Y.M., A.N. and K.T.; formal analysis, Y.M., A.N. and K.T.; investigation, M.A., T.S. and H.H.; resources, Y.M., A.N. and K.T.; data curation, Y.M., A.N. and K.T.; writing-original draft preparation, Y.M., A.N., Y.Y. and K.T.; writing-review and editing, M.A., T.S., H.H., Y.M., A.N., Y.Y. and K.T.; visualization, Y.M. and Y.Y.; supervision, K.T.; project administration, Y.M.; funding acquisition, K.T. GlaxoSmithKline K.K authors contributed to the design, execution, interpretation, and writing of the manuscript. All authors have read and agreed to the published version of the manuscript.

Funding: This study (NCT03261167) was funded by GlaxoSmithKline K.K.

Acknowledgments: We would like to express our deepest gratitude to Ryuji Kaji (Director of National Hospital Organization Utano Hospital), who provided us with valuable advice at the initiation of this trial, and the investigators of the medical institutions participating in the trial. List of principal investigators: Toshikazu Ogasawara, Minami-Sapporo Neurosurgical Clinic; Masayuki Hosaka, Sanyudo Rehabilitation Center; Kazuaki Ebihara, Hitachi, Ltd. Hitachinaka General Hospital; Tsuneo Okada, Tsuchiura Kyodo General Hospital; Akito Hayashi, Juntendo University Urayasu Hospital; Masachika Niimi, The Jikei University Kashiwa Hospital; Toru Takekawa, Tokyo Teishin Hospital; Masahiro Abo, The Jikei University Hospital; Shu Watanabe, The Jikei University Daisan Hospital; Kazuo Koizumi, Izumi Memorial Hospital; Toshiaki Furukawa, Tokai University Hachioji Hospital; Marika Tsuboi, Satoshi Miyano, Tokyo General Hospital; Fujiko Sakuma, Yokohama City University Medical Center; Yoshihisa Masakado, Tokai University Hospital; Kenji Sampei, Nishi Yokohama International Hospital; Nobuyuki Kawate, Showa University Fujigaoka Rehabilitation Hospital; Kentaro Ota, National Hospital Organization Niigata Hospital; Toyoaki Miura, Fukui General Clinic; Hiroyoshi Hara, Kikyogahara Hospital; Sangsoo Eun, Koyama Rehabilitation Hospital; Takashi Shigematsu, Kenjiro Kunieda, Seirei Hamamatsu City Rehabilitation Hospital; Hiroki Takeuchi, Higashi Nagoya National Hospital; Tsukasa Tamaru, Kaikokai Rehabilitation Hospital; Kenji Ohyama, Matsubara Tokushukai Hospital; Toshimitsu Suga, Kansai Medical University Medical Center; Isao Sasaki, Ainomiyako Neurosurgery Hospital; Miki Ohgushi, Hyogo Rehabilitation Center Central Hospital; Ken Kouda, Wakayama Medical University Hospital; Yoshiko Morita, Okayama Rehabilitation Hospital; Shigeru Yamori, Brain Attack Center Ota Memorial Hospital; Shinichiro Takeshita, Hiroshima Prefectural Hospital; Ryosuke Miyamoto, Tokushima University Hospital; Hikaru Mizobuchi, Medical Corporation Shinmatsudakai Atago Hospital; Nobuyuki Irie, Fukuoka Rehabilitation Hospital; Toyoko Asami, Saga University Hospital; Kenshi Obaru, Kumamoto Takumadai Rehabilitation Hospital; Junichi Inobe, Medical Corporation Ikeikai Inobe Hospital; Kimiyoshi Arimura, Sanshukai Ookatsu Hospital. All listed authors meet the criteria for authorship set forth by the International Committee for Medical Journal Editors. Editorial support (assembling tables and figures, collating authors comments, copyediting, factchecking and referencing; Elizabeth Engle and Sarah Hummasti) and graphic services were provided by AOIC, LLC, and were funded by GlaxoSmithKline K.K. Funding for journal fees and permissions was provided by GlaxoSmithKline K.K.

Conflicts of Interest: This study (NCT03261167) was conducted with a research fund from GlaxoSmithKline K.K., in which Masahiro Abo, Takashi Shigematsu and Hiroyoshi Hara participated as principal investigator and their institutions received funding in relation to this work. Yasuko Matsuda, Akinori Nimura, Yoshiyuki Yamashita and Kaoru Takahashi are employees of GlaxoSmithKline K.K. OnabotulinumtoxinA was provided by Allergan plc.

\section{References}

1. Lance, J.W. Symposium synopsis. In Spasticity: Disordered Motor Control; Feldman, R.G., Young, R.R., Koella, W.P., Eds.; Year Book Medical Publishers: Chicago, IL, USA, 1980; pp. 485-494.

2. Doan, Q.V.; Brashear, A.; Gillard, P.J.; Varon, S.F.; Vandenburgh, A.M.; Turkel, C.C.; Elovic, E.P. Relationship between disability and health-related quality of life and caregiver burden in patients with upper limb poststroke spasticity. PM R 2012, 4, 4-10. [CrossRef]

3. Esquenazi, A.; Novak, I.; Sheean, G.; Singer, B.J.; Ward, A.B. International consensus statement for the use of botulinum toxin treatment in adults and children with neurological impairments: Introduction. Eur. J. Neurol. 2010, 17 (Suppl. 2), 1-8. [CrossRef] 
4. Sheean, G.; Lannin, N.A.; Turner-Stokes, L.; Rawicki, B.; Snow, B.J. Botulinum toxin assessment, intervention and after-care for upper limb hypertonicity in adults: International consensus statement. Eur. J. Neurol. 2010, 17 (Suppl. 2), 74-93. [CrossRef]

5. Brin, M.F.; James, C.; Maltman, J. Botulinum toxin type A products are not interchangeable: A review of the evidence. Biologics 2014, 8, 227-241. [CrossRef]

6. Kaji, R.; Osako, Y.; Suyama, K.; Maeda, T.; Uechi, Y.; Iwasaki, M. Botulinum toxin type A in post-stroke upper limb spasticity. Curr. Med. Res. Opin. 2010, 26, 1983-1992. [CrossRef]

7. Honaga, K.; Masakado, Y.; Oki, T.; Hirabara, Y.; Fujiwara, T.; Ota, T.; Kimura, A.; Liu, M. Associated reaction and spasticity among patients with stroke. Am. J. Phys. Med. Rehabil. 2007, 86, 656-661. [CrossRef]

8. Schramm, A.; Ndayisaba, J.P.; auf dem Brinke, M.; Hecht, M.; Herrmann, C.; Huber, M.; Lobsien, E.; Mehnert, S.; Reuter, I.; Stenner, A.; et al. Spasticity treatment with onabotulinumtoxin A: Data from a prospective German real-life patient registry. J. Neural Transm. 2014, 121, 521-530. [CrossRef]

9. Turner-Stokes, L.; Fheodoroff, K.; Jacinto, J.; Maisonobe, P. Results from the Upper Limb International Spasticity Study-II (ULIS-II): A large, international, prospective cohort study investigating practice and goal attainment following treatment with botulinum toxin A in real-life clinical management. BMJ Open 2013, 3, e002771. [CrossRef]

10. Genet, F.; Schnitzler, A.; Droz-Bartholet, F.; Salga, M.; Tatu, L.; Debaud, C.; Denormandie, P.; Parratte, B. Successive motor nerve blocks to identify the muscles causing a spasticity pattern: Example of the arm flexion pattern. J. Anat. 2017, 230, 106-116. [CrossRef]

11. Şengül, İ.; Aşkin, A.; Bayram, K.; Tosun, A. Assessment of post-stroke elbow flexor spasticity in different forearm positions. Somatosens. Mot. Res. 2018, 35, 218-222. [CrossRef]

12. Brashear, A.; Zafonte, R.; Corcoran, M.; Galvez-Jimenez, N.; Gracies, J.M.; Gordon, M.F.; McAfee, A.; Ruffing, K.; Thompson, B.; Williams, M.; et al. Inter- and intrarater reliability of the Ashworth Scale and the Disability Assessment Scale in patients with upper-limb poststroke spasticity. Arch. Phys. Med. Rehabil. 2002, 83, 1349-1354. [CrossRef]

13. Esquenazi, A.; Mayer, N.; Garreta, R. Influence of botulinum toxin type A treatment of elbow flexor spasticity on hemiparetic gait. Am. J. Phys. Med. Rehabil. 2008, 87, 305-310. [CrossRef]

14. Hirsch, M.A.; Westhoff, B.; Toole, T.; Haupenthal, S.; Krauspe, R.; Hefter, H. Association between botulinum toxin injection into the arm and changes in gait in adults after stroke. Mov. Disord. 2005, 20, 1014-1020. [CrossRef]

15. Brin, M.F. Dosing, administration, and a treatment algorithm for use of botulinum toxin A for adult-onset spasticity. Muscle Nerve 1997, 20 (Suppl. 6), S208-S220.

16. Bohannon, R.W.; Smith, M.B. Interrater reliability of a modified Ashworth scale of muscle spasticity. Phys. Ther. 1987, 67, 206-207. [CrossRef]

17. Patel, A.; Geis, C.; Alter, K.; Pan, G.; Thorpe, A.; James, L.; Dimitrova, R. Safety and efficacy of high-dose onabotulinumtoxinA for post-stroke upper limb spasticity: Results of a double-blind, placebo-controlled trial. Neurology 2017, 88 (Suppl. 16), P3.027.

(C) 2020 by the authors. Licensee MDPI, Basel, Switzerland. This article is an open access article distributed under the terms and conditions of the Creative Commons Attribution (CC BY) license (http://creativecommons.org/licenses/by/4.0/). 